\title{
Preservação digital em instituições de ensino superior no Brasil: aspectos, estratégias e políticas
}

Digital preservation in Brazilian higher education institutions: characteristics, strategies and policies

\author{
José Carlos Abbud GRÁcıo (1) e Bárbara FADEL (2)
}

Faculdade de Filosofia e Ciências - UNESP, - Av. Hyginio Muzzi Filho, 737 Marília - São Paulo - Brasil -CEP: 17525-900. (1) gracio@marilia.unesp.br (2) bafadel@terra.com.br

\begin{abstract}
Resumen
Se analizan los principales aspectos, estrategias y políticas relacionados con la preservación digital en las instituciones de enseñanza superior en Brasil. En ese panorama, surgen nuevas amenazas y desafíos: hardware, software, avances en las tecnologías de acceso a la información digital, la cantidad de información digital, cambios en el formato de los archivos, falta de una cultura de preservación, y el coste elevado para las acciones de preservación digital. Sen segundo lugar, se analizan los principales aspectos que afectan a la preservación digital: objetivos de la institución, modelo, responsabilidades, recursos financieros, selección, derechos autorales, autenticidad, infraestructura tecnológica, compendios digitales, equipo multidisciplinar, estrategias de preservación, soporte, metadatos, legislaciones y leyes específicas, y políticas de preservación digital. Como la preservación digital está directamente relacionada con las nuevas tecnologías de información y comunicación, cualquier política o estrategia debe ser dinámica y revisada periódicamente con el objetivo de acompañar los constantes cambios y avances de esas tecnologías. Se observa que la preservación digital debe estar incluida en los objetivos de la institución, con organización administrativa, recursos financieros, equipo multidisciplinar especializado, recursos tecnológicos; y que debería formar parte de cualquier proyecto de tecnología de la información.
\end{abstract}

Palabras clave: Preservación digital. Política de preservación digital. Institución de enseñanza superior. Gestión de la información digital. Metadatos. Estrategias de preservación. Cultura de la organización.

\section{Introdução}

A invenção dos computadores, o surgimento da Internet, a globalização e as constantes mudanças e avanços nas tecnologias de informação e comunicação (TICs) têm afetado a rotina diária das pessoas e das instituições, trazendo gran-

\begin{abstract}
The main aspects, strategies and policies related to the digital preservation in the superior teaching institutions in Brazil are analyzed. In this setting, there are new threats and challenges to be faced, such as: hardware, software, advances in technologies of access to digital information, the amount of digital information, changes in the format of files, the lack of a preservation culture, the high cost for the actions of digital information preservation. The following aspects were observed to affect digital preservation: the goals of the institution, model, responsibilities, financial resources, selection, copyrights, authenticity, technological infra-structure, digital repositories, multidisciplinary team, preservation strategies, support, metadata, legislations and specific laws, preservation policies. Since digital preservation is directly related to new information and communication technologies, any policy or strategy must be dynamic and reviewed periodically in order to follow the constant changes and advances of these technologies. It is concluded that the digital preservation should be added to the strategic objectives of the institution, with addequate administrative organization, financial resources, specialized multidisciplinary team, and technological resources; and that it should be part of any information technology project.
\end{abstract}

Keywords: Digital preservation. Digital preservation policy. Higher education institution. Digital information management. Metadata. Preservation strategies. Organizational culture.

des benefícios e também uma nova cultura. Nesse cenário é cada vez maior a utilização e a dependência das pessoas e das instituições em relação à informação armazenada em meio digital.

Assim, nesse universo digital, surgem problemas a serem enfrentados, principalmente relati- 
vos à obsolescência tecnológica dos equipamentos utilizados (hardware), dos programas de computador (software) e dos suportes de armazenamento.

Essa obsolescência tecnológica nos leva à necessidade de se buscar soluções para a recuperação, no futuro, dessas informações armazenadas em meio digital. Nesse contexto, surge a necessidade da preservação de um novo tipo de patrimônio, a informação digital, armazenada nos computadores e nos suportes digitais, e com ela uma nova área de pesquisa, a preservação digital.

Pesquisas têm demonstrado uma preocupação cada vez maior das instituições como o problema da preservação e do arquivamento digital, mas também mostram que a maioria dessas instituições ainda não tem planos de ações efetivas para tratar o problema (National Library of Australia, 2006).

As instituições de ensino superior (IES) possuem em suas instâncias uma variedade e uma quantidade de informações armazenadas em meio digital, oriundas tanto da produção acadêmica de seus docentes e discentes como das atividades administrativas. Da mesma forma que qualquer instituição, as IES devem ter a preocupação de preservar essa informação digital para que a mesma permaneça acessível por um longo período de tempo.

\section{Objetivos}

Essa pesquisa tem por objetivo estudar os aspectos que envolvem a preservação digital e a forma como as IES devem tratar essa questão para a preservação de suas informações armazenadas em meio digital.

Foram realizadas análises dos desafios que envolvem a preservação digital, mapeando os principais aspectos e estratégias a serem tratados na implementação de uma política de preservação digital nas IES.

\section{Referencial teórico}

A informação pode ser definida como "conjuntos significantes com a competência e a intenção de gerar conhecimento no indivíduo, em seu grupo e na sociedade" (Barreto, 1999). Essa definição mostra a relação estabelecida entre a informação e sua capacidade de gerar conhecimento.

A informação digital poder ser definida como um tipo de informação, com os mesmos objetivos, mas cuja especificidade se refere a suas formas de produção, organização, administração, distribuição e preservação, bem como quanto aos suportes de armazenamento. Podemos definir o suporte de armazenamento como o meio físico no qual a informação digital está armazenada.

Ferreira (2006) denomina as informações armazenadas em meio digital de objetos digitais, que podem ser definidos como todo e qualquer objeto de informação que possa ser representado através de uma seqüência de dígitos binários, como por exemplo, textos científicos, bancos de dados, fotos digitais, vídeos, páginas Web, software, etc.

Surgida no final do século $X X$, a informação digital deixou como um dos grandes desafios para o século $X X I$ a preservação desse novo tipo de informação.

Ferreira (2006) define preservação digital como a capacidade de garantir que a informação digital permaneça acessível e com qualidade de autenticidade para que possa, no futuro, ser interpretada numa plataforma tecnológica diferente daquela utilizada em sua criação.

Para Arellano (2004), a preservação digital compreende mecanismos que permitem o armazenamento em repositórios de dados digitais que garantam a perenidade dos seus conteúdos, integrando a preservação física, lógica e intelectual dos objetos digitais.

A National Library of Australia (2008) entende a preservação digital como "o processo envolvido na manutenção, e se necessário, no restabelecimento da acessibilidade para os recursos de informação digital"

Portanto, podemos observar que os objetivos a serem alcançados na preservação digital são os mesmos envolvidos na preservação da informação de modo geral, ou seja, garantir que essa informação possa ser recuperada ao longo do tempo com garantia de autenticidade, mesmo com as mudanças constantes nas tecnologias e na gestão das instituições.

Nesse ambiente de mudanças, muitas são as ameaças e os desafios a serem enfrentados para que essa informação possa ser preservada para o acesso ao longo do tempo. Entre esses desafios e ameaças, podemos citar:

- obsolescência do hardware e do software, que com os avanços tecnológicos, tornam-se ultrapassados muito rapidamente;

- mudanças e avanços nas tecnologias de acesso à informação digital, causadas principalmente pelo surgimento da internet;

- a explosão da quantidade de informação armazenada em meio digital, que cresce a cada 
dia, substituindo os meios de armazenamento tradicionais;

- as mudanças no formato dos arquivos e das mídias de armazenamento;

- a falta de uma cultura de preservação por parte dos criadores da informação digital e por parte das instituições responsáveis em preservar essa informação;

- o custo elevado para as ações de preservação digital e necessidade de recursos financeiros permanentes no orçamento das instituições.

\section{Metodologia}

O universo desta pesquisa são as IES, por possuírem um conjunto extenso de informações digitais.

O método de pesquisa bibliográfica foi utilizado para realizar um levantamento junto às principais revistas nacionais e internacionais com $o$ objetivo de identificar como as IES estão tratando a questão da preservação digital e de levantar os principais aspectos e estratégias relacionadas a ela.

Através do levantamento bibliográfico, também se buscou identificar os principais projetos implantados e/ou em desenvolvimento.

\section{Preservação digital: aspectos e estratégias}

Observa-se no levantamento realizado que as iniciativas de preservação digital nas IES no Brasil estão mais presentes nas bibliotecas universitárias, mais especificamente nas atividades de gerenciamento de bibliotecas digitais.

A comunidade acadêmica tem demonstrado, a cada dia, mais interesse na busca e recuperação de informações digitais e também na disponibilização de sua produção em meio digital.

Nesse contexto, a preservação digital nas IES torna-se também um instrumento importante de preservação da produção científica.

Além dos aspectos técnicos, a preservação digital nas IES depende de questões institucionais, tais como a mudança periódica de direção, da administração, de objetivos e de fontes de financiamento. Observa-se ainda que as IES no Brasil não possuem políticas de preservação digital definida em suas instâncias superiores.

Vários são os aspectos que envolvem a preservação digital em uma instituição, e a definição de uma política de preservação digital que aten- da às necessidades da mesma depende de como esses aspectos são tratados.

A seguir citamos os principais aspectos e estratégias envolvidos na preservação digital:

\subsection{Objetivos da instituição}

Para a definição de quais os tipos de informação institucional devem ser preservadas, é importante definir claramente os objetivos da instituição.

A partir da definição dos tipos de informação digital a serem preservados, é possível definir os princípios a serem adotados na instituição para as atividades de preservação digital, princípios esses que devem seguir leis federais, estaduais e municipais, além de legislações próprias das IES.

\subsection{Seleção}

É necessário definir o que deve ser preservado, ou seja, selecionar as informações digitais a serem preservadas de acordo com os objetivos da instituição e as necessidades da comunidade acadêmica da IES. Essa é uma questão delicada, pois a instituição pode definir que toda informação digital é importante. É uma utopia imaginar que é possível preservar toda informação digital produzida por uma instituição.

A definição de prioridades referente ao que deve ser preservado é desejável juntamente com o processo de seleção, pois as atividades de preservação digital podem sofrer atrasos ou restrições, devido ao grande volume de informações.

\subsection{Modelos, padrões e iniciativas}

Como o desafio da preservação digital é global, recomenda-se a adoção de padrões e modelos já utilizados e testados por instituições que trabalham com a preservação digital, garantindo assim uma maior possibilidade de continuidade do padrão adotado e a possibilidade da troca de informações entre instituições (interoperabilidade). Um dos modelos atualmente recomendados é o Open Archival Information Systems (OAIS), desenvolvido pelo Consultative Committee for Space Data Systems (CCSDS) pela NA$\mathrm{SA}$. Este modelo é uma norma ISO com o $\mathrm{n}^{\circ}$ 14721:2002 que descreve um enquadramento conceitual para um repositório digital genérico, aberto a todas as comunidades e com garantias de confiabilidade.

O desafio da preservação digital é grande e está sendo enfrentado por algumas instituições através de diversas iniciativas de projetos corporativos, em nível mundial, na busca de soluç- 
ões, principalmente para as informações relacionadas ao desenvolvimento científico e tecnológico de seus países de origem.

Como exemplos, podemos citar: projeto ERPANET na Europa; projeto "Preserving Access to Digital Information" (PADI) da Biblioteca Nacional da Austrália, que define a direção que a biblioteca pretende tomar para preservar suas coleções digitais; projeto "National Digital Information Infrastructure and Preservation Program" (NDIIPP), um dos maiores projetos de preservação digital dos Estados Unidos; e o Digital Preservation Europe (DPE) criado na Europa em 2006, com o objetivo de promover a colaboração entre as iniciativas de preservação digital

No Brasil, uma das iniciativas é a "Carta para a Preservação do Patrimônio Arquivístico Digital" do Conselho Nacional de Arquivos (Conarq).

\subsection{Responsabilidades}

$\mathrm{Na}$ literatura, podemos observar que é consenso que a preservação digital é responsabilidade do criador do objeto digital e também da instituição na qual esse objeto está disponível. Portanto, a instituição deve ter claro quais são as atividades a serem desenvolvidas no processo de preservação e os responsáveis por cada uma delas.

\subsection{Recursos financeiros}

A preservação digital é uma atividade que requer disponibilidade de recursos financeiros, pois envolve investimentos permanentes em tecnologia, infra-estrutura e capacitação de pessoal, ou seja, preservar tem um custo financeiro alto e a longo prazo.

É necessária uma política permanente de investimento por parte da instituição que garanta atualizações dos equipamentos e do software, capacitação da equipe de trabalho, investimentos em infra-estrutura e investimentos nos procedimentos de preservação. Essa política irá garantir que mesmo na mudança do gestor da IES, o processo de preservação permaneça como uma das prioridades da instituição.

\subsection{Autenticidade}

Outra questão importante da preservação digital é o conceito de autenticidade, que pode ser entendido como a capacidade de identificar elementos que permitam definir se um objeto é autêntico ou não. Para isso, é importante a definição das propriedades desse objeto digital que deverão ser mantidas e preservadas para que o mesmo possa ser considerado autêntico, influ- enciando também diretamente na forma com esse deverá ser preservado (Ferreira, 2006).

O usuário que busca uma informação digital precisa ter a certeza que essa informação é autêntica, ou seja, as instituições precisam garantir a autenticidade da mesma através de estratégias bem definidas e que dêem ao usuário segurança quando este for utilizar a informação.

Assim como o usuário que busca a informação digital, o responsável por esse objeto digital também tem a necessidade que a instituição garanta que aquele trabalho desenvolvido por ele permaneça da forma como foi criado.

\subsection{Direitos autorais}

Como a preservação digital implica muitas vezes em reproduzir o objeto digital, o problema dos direitos autorais é um aspecto importante, pois o material original é protegido pela lei dos Direitos Autorais. Portanto, qualquer estratégia deve estar amparada em leis que respaldem a instituição e garantam ao autor do objeto digital a propriedade intelectual do mesmo.

\subsection{Infraestrutura tecnológica}

A infraestrutura tecnológica é fundamental para atender adequadamente cada fase da vida de um objeto digital e garantir que o mesmo esteja disponível para busca, recuperação e acesso. Essa infraestrutura tem que garantir ao objeto digital que está sendo preservado principalmente sua integridade, segurança, o correto armazenamento, políticas de backup e prevenção/recuperação de desastres.

Nesse sentido, a capacitação permanente dos profissionais responsáveis por essas atividades é fundamental, pois da mesma forma que as TICs, a infraestrutura passa por constantes avanços e, consequentemente, pela necessidade de readequação a essas novas tecnologias.

\subsection{Repositórios digitais}

O desenvolvimento de repositórios digitais emergiu como uma nova estratégia de gerenciamento e divulgação da produção científica no começo do século XXI. Com o aumento da quantidade de material digital nas universidades e o surgimento de software (livre ou proprietário) de tratamento dessa informação, a implementação de repositórios digitais nas universidades passou a ser uma ferramenta importante para a divulgação da produção científica.

Da mesma forma que a preservação digital exige investimentos a longo prazo, os repositórios 
digitais também necessitam de planejamento e investimentos em infraestrutura por longos períodos de tempo. Esse planejamento deve incluir normas e políticas bem definidas.

Nesse contexto, surge a definição de repositórios de dados sustentáveis, ou seja, repositórios capazes de gerenciar e manter os dados disponíveis ao longo do tempo. Como o número de repositórios cresce a cada dia, vários trabalhos no campo da auditoria e certificação têm surgido com o objetivo de desenvolver critérios de auditoria em repositórios digitais e assim distinguir aqueles que podem ser considerados sustentáveis ou não.

\subsection{Equipe multidisciplinar}

Com o crescente aumento das informações digitais e a necessidade da preservação das mesmas, surge a necessidade das instituições montarem equipe multidisciplinar com profissionais de várias áreas para o tratamento desse tipo de informação. Além dos bibliotecários e arquivistas, podemos citar como necessários às atividade de preservação digital profissionais das áreas de informática, sistemas de informação, direito, administração, engenharia, docentes da IES e outros que a instituição julgar necessários.

\subsection{Estratégias de preservação}

É necessário que as IES adotem estratégias de preservação bem definidas para cada tipo de objeto digital, avaliando-as periodicamente para determinar qual a melhor estratégia a ser implementada de acordo com as tecnologias existentes naquele momento.

As estratégias de preservação estão baseadas em dois tipos de conservação do objeto digital:

\subsubsection{Conservar o objeto digital no seu forma- to original através das técnicas de:}

- Refrescamento: transferir a informação de um objeto físico de armazenamento (suporte) para outro mais atual, antes que o primeiro deteriore;

- Emulação: técnica de criar um ambiente tecnológico que emule o ambiente original do objeto digital; mais relevante na preservação de aplicações de software, como por exemplo, jogos de computador.

\subsubsection{Conservar o conteúdo intelectual do} objeto digital, através da:

- Migração: técnica que consiste em transferir periodicamente um objeto digital de uma tec- nologia de hardware e/ou software para outra mais atual.

Existem também propostas de criação de diretórios centralizados de informação técnica sobre os formatos digitais, que permitam, por exemplo, disponibilizar ferramentas que ajudem a identificar o formato de um objeto.

\subsection{Suporte}

É necessário que as IES utilizem meios de suporte que permitam, além da preservação do objeto digital, sua busca e recuperação.

Os suportes são meios de armazenamento que possuem uma vida útil e que, portanto necessitam de controle para que sejam realizadas periodicamente atividades de refrescamento, além do controle de variáveis ambientais que minimizem riscos de deterioração precoce do suporte de armazenamento.

\subsection{Metadados}

Metadados relacionados a informações armazenadas em meio digital podem ser definidos como um conjunto de elementos que descrevem as informações contidas em um recurso digital, com o objetivo de possibilitar sua busca, recuperação e preservação. Portanto, são essenciais não somente para a descrição do conteúdo de um objeto digital, mas também para as atividades de preservação do mesmo.

Esses elementos devem conter informações descritivas (ex: título, autor), temáticas (ex: palavras-chave) e de preservação (ex: formato). Existem vários padrões existentes, entre eles alguns mais simples e genéricos, mas amplamente utilizados, como o Dublin Core e o Resource Description Format (RDF) e outros mais complexos e sofisticados como o Metadata Encoding and Transmission Standards (METS) e o Encoded Archival Description (EAD). Os metadados de preservação devem acompanhar todo o ciclo de vida do recurso e apoiar todo o processo de preservação digital ao longo do tempo, registrando todas as estratégias aplicadas e as mudanças ocorridas.

Todos esses aspectos e estratégias, que norteiam a preservação digital, apontam para a necessidade de implementação de políticas de preservação digital nas IES.

\section{Políticas de preservação digital}

O termo política deve ser entendido como um conjunto de objetivos que dão forma a um programa de ação gerencial ou administrativa e condicionam sua execução. 
A política de preservação digital deve ter como objetivo garantir que as instituições tenham um planejamento e estratégias bem definidas para o armazenamento e uso de recursos de informação digital para longos períodos de tempo. Essa política deve garantir a continuidade do processo de preservação digital.

Como a preservação digital está diretamente relacionada às novas TICs, qualquer política de preservação digital deve ser dinâmica e revista periodicamente com o objetivo de acompanhar as constantes mudanças e avanços dessas tecnologias.

\section{Considerações finais}

A preservação digital deve estar inserida nos objetivos da IES e nos projetos de Tecnologia da Informação (TI), com organização administrativa, recursos financeiros, equipe multidisciplinar especializada e recursos tecnológicos. A implementação de políticas de preservação digital é a forma de garantir que esses objetivos sejam alcançados.

As IES devem criar uma Comissão Permanente de Preservação Digital, multidisciplinar, que tenha como responsabilidades definir uma política de preservação digital e apontar os procedimentos e estratégias adequados para a instituição.

Como as mudanças são constantes, sejam elas tecnológicas ou de gestão da instituição, é imprescindível que essa comissão realize constantes avaliações das estratégias adotadas, e, quando necessário, mudanças nas políticas e estratégias.

A colaboração entre as instituições na busca por soluções é um dos caminhos que podem agilizar e contribuir na resolução dos problemas, pois os custos da preservação digital são altos, assim como os conhecimentos exigidos nas atividades de preservação.

O desafio da preservação digital é grande, pois enquanto as instituições estão discutindo suas políticas e estratégias, muita informação digital está sendo perdida, tanto por falta de conhecimento, como por falta de cultura de preservação das pessoas e das instituições.

É necessário que as IES insiram em sua cultura organizacional a cultura da preservação digital.

\section{Referências}

Arellano, Miguel Angel (2004). Preservação de documentos digitais. /I Ciência da Informação. 33:2 (2004). http://www.scielo.br/pdf/ci/v33n2/a02v33n2.pdf (200701-04).
Barreto, Aldo Albuquerque (1999). A oferta e a demanda da informação: condições técnicas, econômicas e políticas. // Ciência da Informação. 28:2 (1999) 168-173.

Boeres, Sônia A. de Assis; Arellano, Miguel Angel Mardero (2005). Políticas e estratégias de preservação digitais de documentos. // Anais do VI Encontro de Ciência da Informação - CINFORM, Salvador, BA, 2005. http://dici.ibict.br/archive/00000263/01/Preserva\%C3\%A 7\%C3\%A3o_VI_CINFORM.pdf (2007-01-04).

UNESCO (2003). Carta sobre la preservación del patrimonio digital. 2003. http://portal.unesco.org/es/ev.php URL_ID=17721\&URL_DO=DO_TOPIC\&URL_SECTION $=201 . h t m$ (2007-01-17).

CONARQ (2004). Carta para a Preservação do Patrimônio Arquivístico Digital. Rio de Janeiro: CONARQ, 2004. http://www.conarq.arquivonacional. http://gov.br/Media/ publicacoes/cartapreservpatrimarqdigitalconarq2004.pdf (2007-01-17)

Ferreira, Miguel (2006). Introdução à preservação digital Conceitos, estratégias e actuais consensos. Guimarães, Portugal: Escola de Engenharia da Universidade do Minho, 2006. https://repositorium.sdum.uminho.pt/bitstrea m/1822/5820/1 /livro.pdf (2007-01-17).

National Library of Australia (2006). Recent developments in digital archiving and preservation. 2006. http://www.nla.gov.au/nla/staffpaper/2006/documents/pg atenby_CDNL.pdf (2009-03-25).

National Library of Australia (2008). Digital preservation policy. 2008. http://www.nla.gov.au/policy/digpres.html (2008-05-15).

OCLC (2006). OCLC Digital Archive Preservation Policy and Supporting Documentation. 2006. http://www.oclc.org/su pport/documentation/digitalarchive/preservationpolicy. pdf (2009-02-25).

Rothenberg, Jeff (1995). Ensuring the longevity of digital documents. // Scientific American. (Jan. 1995) 24-29.

Saramago, Maria de Lurdes (2003). Metadados para preservação digital e aplicação do modelo OAIS. 2003. http://www.unicamp.br/siarq/doc_eletronico/ metadados.pdf (2009-04-22).

Thomaz, Katia P.; Soares, Antônio José (2004). A preservação digital e o modelo de referência Open Archival Information system (OAIS). // DataGramaZero. 5:1 (Fev. 2004). http://datagramazero.org.br/fev04/index.htm (2008-08-04). 